\title{
Teaching Biochemistry and Molecular Biology With Virtual Reality - Lesson Creation and Student Response
}

Heather A. Coan

Western Carolina University

Geoff Goehle

Pennsylvania State University (current institution)

Western Carolina University

Robert T. Youker

Western Carolina University

\begin{abstract}
The commercialization of virtual reality (VR) hardware has enabled the use of VR as an educational tool. We describe how a VR platform was used to create molecular visualizations using standard PDB files with the purpose of delivering biochemistry and cellular biology lessons for undergraduates. Specifically, we describe two new software modules, one for rendering molecules generated from PDB files and one for creating interactive lectures. These modules were used to create two VR labs, one on DNA and collagen and the other on hemoglobin. We then describe how these labs were delivered, along with surveys and quizzes, to over 100 students in undergraduate biology courses. Student response to the labs are found to be extremely positive and the labs themselves are found to satisfy their educational objectives.
\end{abstract}

\section{Introduction}

In recent years there has been an explosion in the availability of high-grade consumer virtual Reality (VR) equipment. These new VR devices, such as the HTC Vive and the Oculus Rift, open up the possibility of using VR visualizations in educational settings. This is especially exciting in the case of biology education where there is a need for intuitive and high-quality 3D visualizations of molecular structures. Understanding three-dimensional molecular configuration is especially important for the study of proteins and large biomolecules and is a longstanding challenge in 
biology education (Lam \& Siu, 2017). Virtual Reality has the potential to render standard molecular visualizations "realistically." It can enable students to manipulate proteins as if they were physical models, while also allowing them to dynamically switch between different visualizations such as ball and stick, ribbon and wire, or surface representations.

While there is a lot of potential in using VR as an educational tool, there are also significant barriers. First, the standard tools for creating VR models and environments require a substantial technical background. There is a paucity of easy to use tools to allow instructors to create high quality VR protein models and even fewer tools for assembling these models into a lab or lesson (Borrel \& Fourches, 2017). While there have been some studies involving VR and biology education (Garcia-Bonete, Jensen, \& Katona, 2019; Garzón, Magrini, \& Galembeck, 2017; Johnston et al., 2018; Kaphingst et al., 2009; Mikropoulos, Katsikis, Nikolou, \& Tsakalis, 2003), there are still a great many open questions. For example, quality VR hardware is still somewhat expensive and can be cumbersome to use in a classroom setting. It is unclear the best way to deliver VR experiences that produce satisfactory educational results and are also scalable to large class sizes. This paper aims to help address these issues by presenting a setup where VR labs can be more easily created using standard resources such as PDB files, traditional PowerPoint slides, and audio recording software. These lessons are then packaged so that they can be delivered to students on an appointment basis outside of class-time. This streamlines the lab creation process and presents a scalable technique for providing VR experiences to students.

In what follows, we first describe two new software modules for the Unity graphics platform. These modules allow instructors without an extensive programming background to use standard PDB files to create dynamic Unity objects capable of displaying multiple visualizations and to use these objects to create interactive slide-based lessons. In the next section we describe the two interactive labs created with these tools. One lab is on DNA and collagen and focuses on helix structures. The second lab is on different forms of hemoglobin. These labs were delivered to over 100 students in the course of a single semester. In addition to participating in the lessons, students completed quizzes and filled out surveys. In the third section we describe the overall procedure for how these labs were delivered to students and in the fourth section we analyze the results of the quizzes and surveys. Overall, we find that the labs meet their educational objectives. Furthermore, student response to the labs is found to be extremely positive. Finally, we end with overall conclusions regarding the implementation of this emerging technology.

\section{Overview of Technology}

The main hardware platform for this project was the HTC Vive. The Vive allows users to move around in a predefined "play area" while wearing a headset and holding controllers. As users move around the play area the Vive creates a virtual three-dimensional environment by rendering slightly different perspectives of a traditional 3D computer environment for each of the user's eyes, as can be seen in Figure 1. The fact that users can walk around the play area normally, rather than being forced to use an alternative locomotion technique such as teleportation helps reduce nausea and physical discomfort. In theory the modules and labs described below could be made to work with any VR system and our choice of the Vive is largely one of preference. Our overall setup is similar to the one used in (Goehle, 2018). 


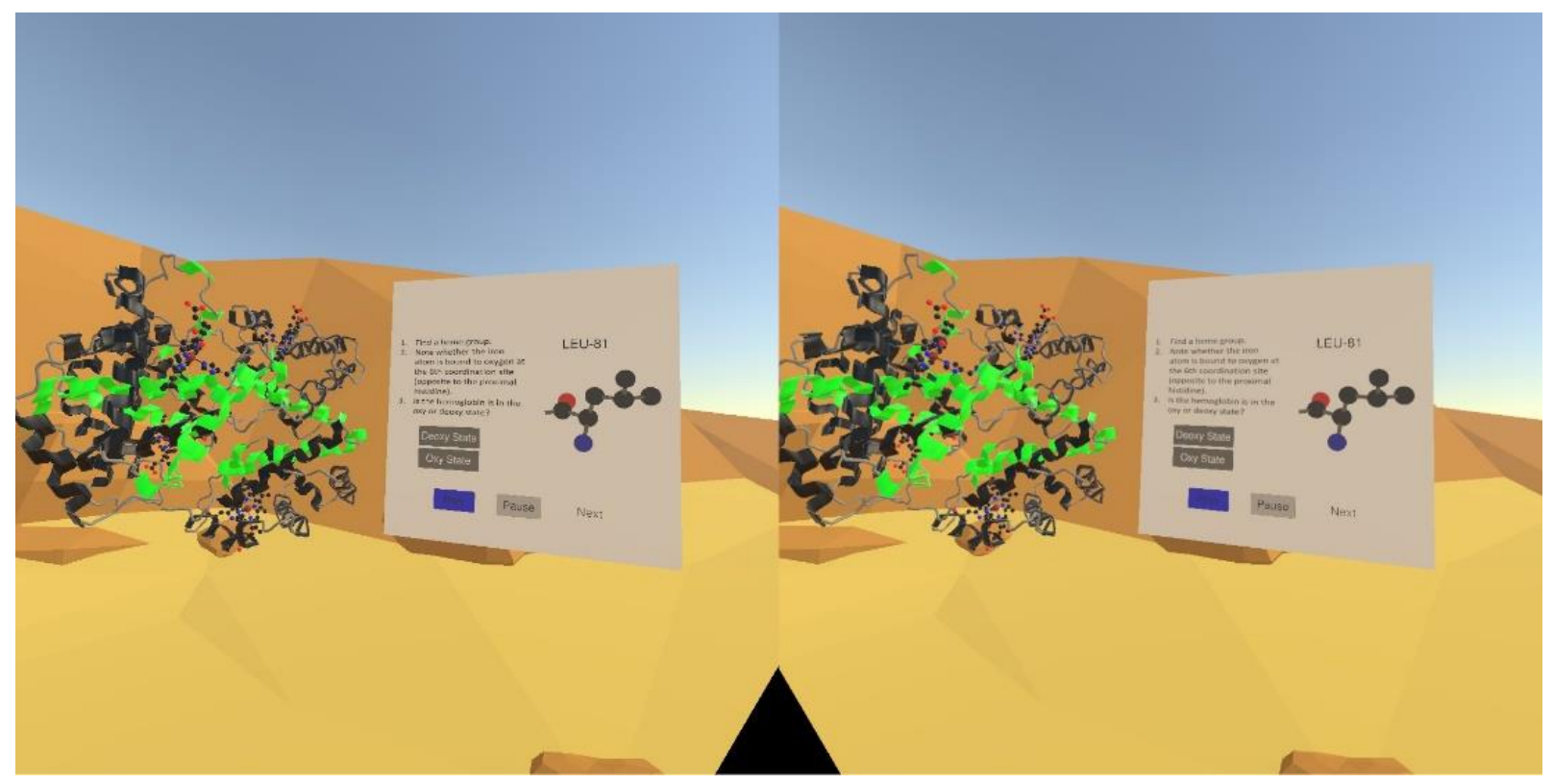

Figure 1: Left Eye/Right Eye VR View. The Vive simulates three dimensional objects by rendering a slightly different perspective for the left and right eyes of the user. Here we see a hemoglobin protein in wire and ribbon mode as well as a lecture slide.

The main software platform for this project was the Unity 3D graphics engine. In general, the tools needed to build VR environments are the same needed to build any 3D computer environment. The Unity graphics engine was chosen because it is relatively inexpensive, and it contains freely available packages which provide the interface to the VR system and the general world environment. There were only two Unity modules which needed to be created from scratch. The first was a PDB importer which could generate Unity objects from PDB files. We needed to create native Unity objects, rather than importing 3D models from external software, because this allows us to make the chemical structure of the molecules an integral part of the object. This in turn makes the objects much more flexible and dynamic. For example, we can selectively display information about individual residues, add and remove bonds, and change the color scheme for the visualization. This would be impossible if the proteins had been imported as fixed models.

The second piece of technology we created was a platform for generating interactive slidebased VR lessons. This package forms a bridge between traditional slide-based lectures, the VR environment and interface, and the protein and DNA Unity objects mentioned above. Standardizing the presentation setup and the interactive components allowed us to create lessons much more quickly than if we crafted each individually using in-engine tools. This section presents a general overview and important features of each package. The packages themselves are available on request from the authors.

\section{Protein Visualizer Package}

This module takes a standard PDB file and creates a hierarchy of Unity objects which allow the corresponding molecule to be visualized using ball and stick, ribbon and wire, and surface visualizations, as can be seen in Figure 2. Specifically, the module parses the PDB file and does the following: 
- Creates individual Unity objects representing the ball and stick model for each residue. Atoms are placed according to their position in the PDB file and are colored and sized according to their element. The bonds are generated algorithmically based off the type of residue and the atom names. The current system supports the standard amino acids and DNA/RNA base pairs.

- Collects the residue objects into chains and adds the chain bonds. At this stage heterogen molecules are also created. Their atoms are placed according to their position in the PDB file and their bond structure is generated from the CONECT elements in the PDB file.

- Creates a wire and ribbon model for each chain. The graphics for the wires and ribbons are programmatically generated and follow the chain backbone. Ribbons are used to represent helices and sheets as defined by the HELIX and SHEET entries in the PDB file. The type of each helix or sheet is included in the object data and is used to color the ribbons.

- Creates a surface representation by generating an isosurface using a probe radius of $1.2 \AA$. The surface is colored according to the electric potential of the charged atoms in the molecule. Only amino acids which are usually charged at physiological $\mathrm{pH}$ were included (Glu, Asp, Arg, Lys, and optionally His). Charge contributed by other sources, such as heterogen atoms, are not considered. Areas colored blue are more negatively charged than average and areas colored red are more positively charged.
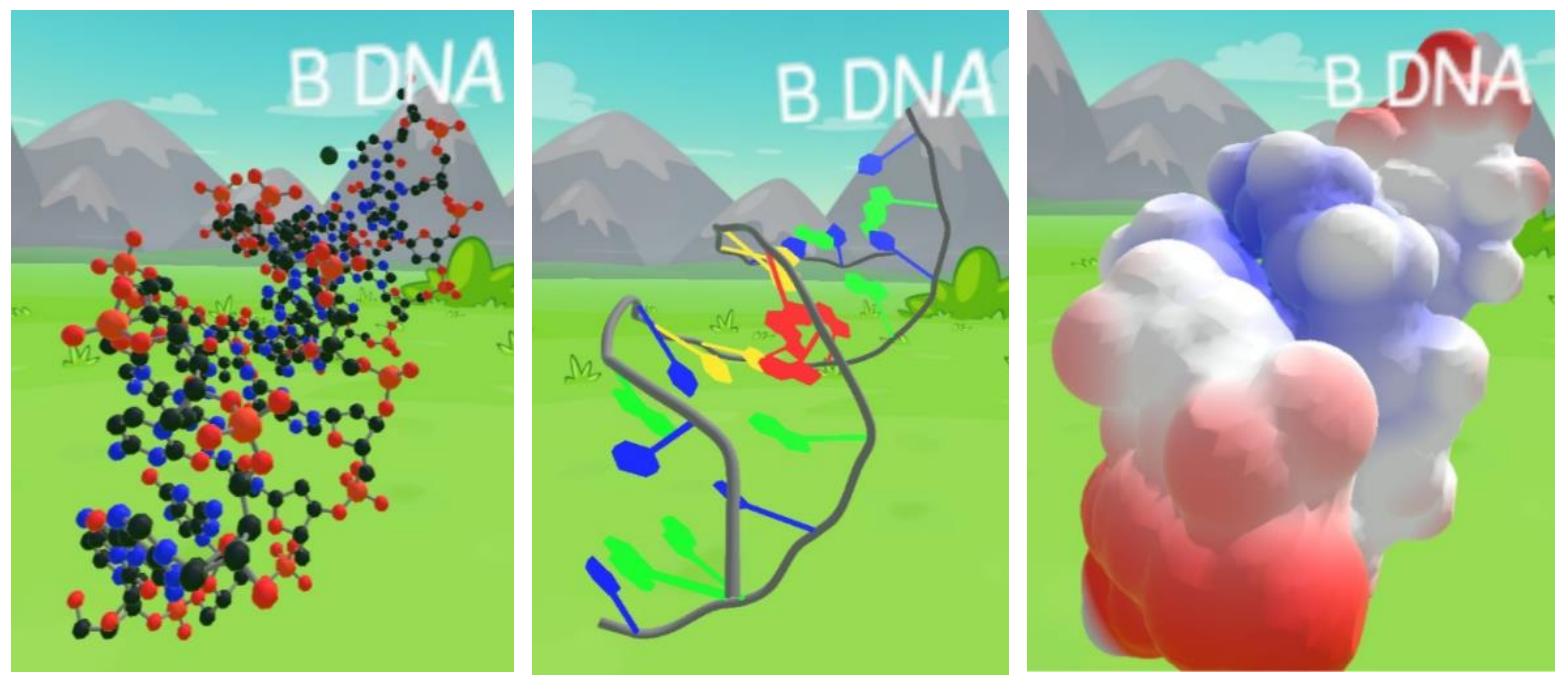

Figure 2: Different Visualizations of B DNA. Here we see three different visualizations of B DNA. The ball and stick representation on the left features scaled atoms colored by element. The wire and ribbon view in the middle has representations of the base pairs. Other molecules feature ribbons to represent sheets and helices. The surface view on the right is colored by the relative electric potential from the molecule. Blue represents regions with more negative potential and red with more positive potential.

The resulting objects are very flexible. Visualizations and color schemes can be altered in the editor using either standard Unity tools or custom editor functions. Properties of the protein objects can also be changed during runtime, allowing users to switch between different visualizations and otherwise dynamically interact with the objects. Each object also contains the 
relevant metadata from the PDB file including residue name and number, helix or sheet type, the title and author of the PDB file, and so on. This data can be accessed and displayed during runtime.

The visualization package was designed to be modular and can be expanded. Advanced features prototyped for this project, but not included in the final lectures were:

- The ability to import PDB files and render or re-render molecule visualizations at runtime. The relevant methods are all written using co-routines to prevent drops in the frame rate.

- The ability to import and display molecule "animations" using a sequence of frame PDB's generated from an external program.

- The ability to animate proteins dynamically using rotations and transforms.

- A feature to add new bonds whenever two atoms are separated by the expected bond distance.

In many respects, this package merely replicates what is done by other, more developed, 3D biomolecule rendering software. This was an intentional part of the design philosophy. Rather than creating custom, highly stylized representations of molecules, we wanted the VR lectures to include models generated from real data that would be familiar to students from other computer visualization settings. However, because we create models using in-engine objects we can provide more interesting interactions to students. It should be noted that this package is largely independent from the rest of the project and can be used to create Unity molecular visualizations for any purpose, VR based or otherwise. While the current system works with a wide range of PDB files, it does not correctly handle all possible modifications and corner cases. More work needs to be done to make the interpreter more robust, as well as to support non-standard residues.

\section{Presentation Platform Package}

The main purpose of this package is to streamline the process of lesson creation. Ideally this would be a fully featured tool to allow instructors not familiar with Unity to create VR lessons. This is beyond the scope of the project, however. Instead this package aims to standardize how lessons are created and presented so that the same basic framework can be used to create multiple lectures. The heart of the package is the "screen stage". This is a region hidden from view of the student where 2D and 3D Unity elements can be placed in front of a camera. The camera view is used to create a Render Texture which is placed on both the student's slide controller, as well as a large "projector screen" in the environment. Using this set up allows us to layer 2D and 3D Unity objects to create interactive slide-based lectures. An example slide, featuring multiple choice questions, is shown in Figure 3.

The slides for a lesson are generated using a program such as PowerPoint and can include an audio track which the student can start and stop at will. They can also include the following interactive elements:

- The slides can dynamically display information about molecules. Students select the modules using one of the VR controllers and the slide will show information such as the residue type and number.

- The slides can have multiple-choice questions. Students must correctly answer the questions to proceed with the lesson. 
- The slides can have a "hide and seek" functionality which requires students to find certain residues in a protein before continuing.

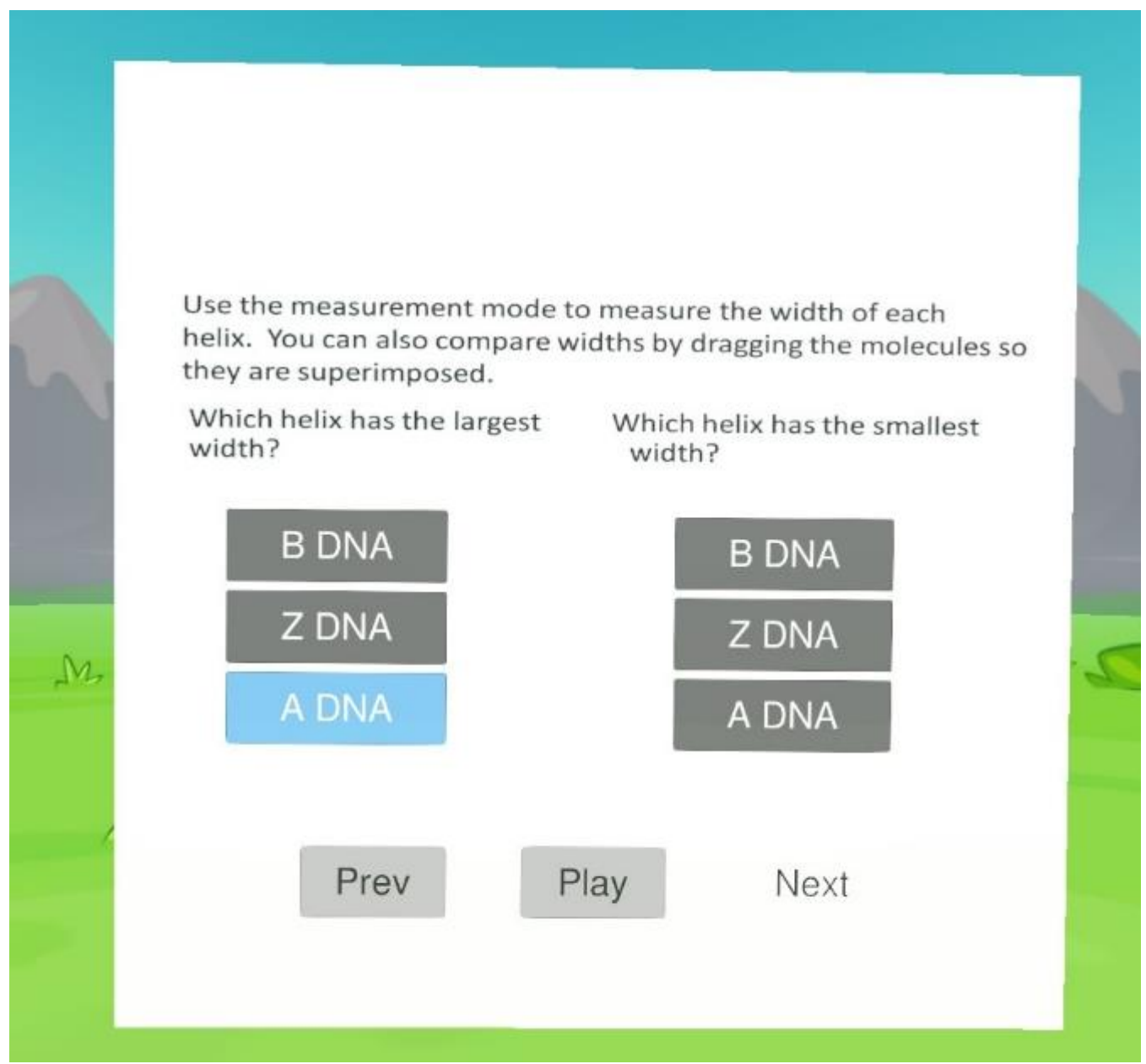

Figure 3: Interactive Slides. The presentation platform supports slides with interactive elements, like the multiple-choice questions featured above. Students must select the correct answers to continue the lecture. Students navigate the slide using one of the VR controllers.

This module also governs the interactivity between the user and the protein objects. Students can use a VR controller to move and rotate the proteins, as well as change their visualization. Generally, students can cycle between ball and stick, wire and ribbon and a surface visualization mode. For transcriptional regulators, students can cycle between the protein and DNA representations separately to create interesting visualizations, and for hemoglobin the heme groups and certain residues were included in the wire visualization to highlight the oxygen bonding sites. Finally, students can use a VR controller to make distance and angle measurements. This allows students to measure the distance between residues and to approximate bond angles. The controller setup can be seen in Figure 4. The controller on the left displays the current slide and allows users to navigate the lesson. The controller on the right allows students to manipulate the molecules, change visualizations, and make measurements. 


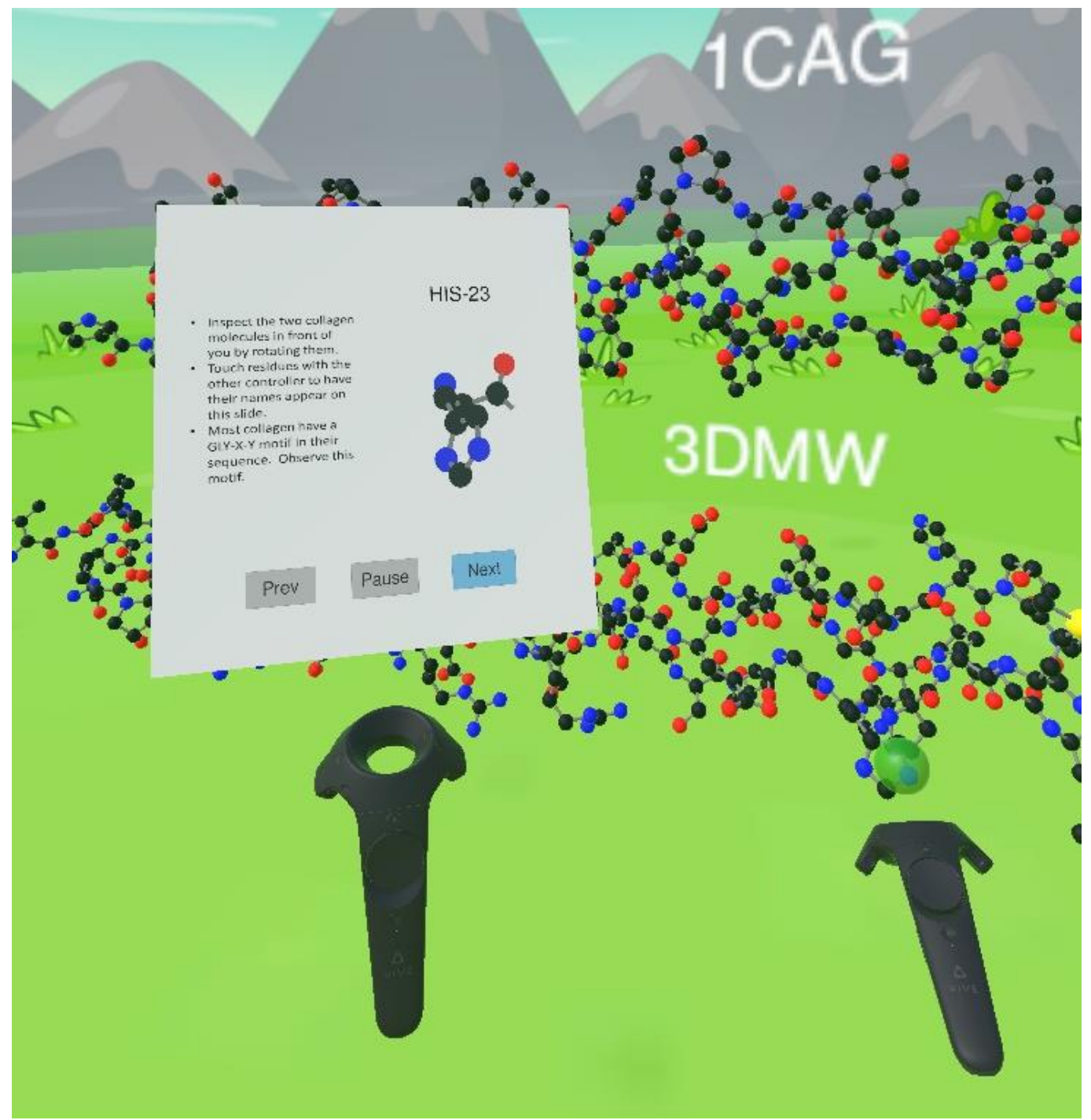

Figure 4 - VR Controls: The two standard Vive controllers served different functions. The left controller displays the current slide and is used to navigate the interactive slide elements. The right controller is used to manipulate the proteins, change visualizations, and make measurements. Here a student is using the right controller to select a residue which is then displayed on the left controller.

Overall, the process for creating a lesson is roughly as follows:

1. An instructor creates a slide-based presentation on a topic. On each slide they make a note of which, if any, molecules should be displayed to the student, what type of visualization should be shown, as well as any multiple choice or interactive elements they wish to include on the slide.

2. The instructor records an audio track to accompany each slide. They send the audio tracks, slides, and associated PDB files to someone proficient with the Unity modules.

3. That person uses specially written editor scripts to create the in-engine slides. Then they use additional scripts to add the interactive elements and audio tracks to the slide objects. 
4. Finally, the person implementing the lecture imports the PDB files and configures their visualization settings. The resulting protein objects are set to only appear on the appropriate slides.

This process was used by two of the authors to create the pair of lessons described in the next section. It is noteworthy that the procedure was robust enough to also be used by an undergraduate to create a third lesson as part of an undergraduate project. Overall the system provides a solid foundation for creating lectures but has room for improvement. It still requires extensive Unity expertise and currently only works with the Vive VR system. Both issues could be fixed with further polish and iteration.

\section{Lesson Descriptions}

The tools described in the previous section were used to create two undergraduate VR labs. The first VR lab is on helix structures in DNA and collagen while the second is on different forms of hemoglobin. Each lab is approximately 20 minutes long and features slides with an accompanying audio track. Taken together the slides and audio tracks are similar to a traditional slide-based lecture. Each slide is accompanied by one or more interactive protein molecules which students can manipulate as described in the previous section. Slides can also contain one or more interactive elements, also as described in the previous section.

\section{Lab 1 - DNA and Collagen}

Undergraduate students are familiar with the characteristic double-helix of DNA. The canonical structure presented in textbooks is of the B-form, which is an averaged structure, first proposed by Watson and Crick and supported by Gosling, and Franklin's experimental data (Portin, 2014). Importantly, DNA can adopt several structural forms in addition to B-form, such as A-form, and Z-form DNA (Ussery, 2002). The width, base pairs-per-turn, and handedness of the helix vary between these three forms. The B and A-form DNA have right-handedness, while Z-form DNA is left-handed. Undergraduate students enrolled in an upper-level cell biology course routinely have difficulty distinguishing the structural changes between the $\mathrm{A}, \mathrm{B}$, and Z-forms of DNA. The changes in DNA structure that occur upon binding of certain transcription factors are also difficult for students to comprehend using 2D representations. In addition to DNA structures, subtle changes in protein secondary structure that can lead to human disease are not readily apparent when using 2D images. The goal of this lesson was to expose students to an interactive and immersive VR environment to determine if it could aid in student understanding of DNA and protein structures.

The DNA and collagen VR lab was divided into three sections: identification of different DNA structures, comparison of wildtype and mutant collagen molecules, and effects of transcription factor binding on DNA structure. In the first section, students were presented with B, Z, and A-form DNA molecules to inspect (PBD ID: 355D [Shui, McFail-Isom, Hu, \& Williams, 1998], 4OCB [Luo, Dauter, \& Dauter, 2014], \& 5MVP [Hardwick, et al., 2017], respectively). The molecules could be displayed in ribbon, ball-and-stick, and surface representations, and students had full control over which representation to display. Students needed to determine the dimensions of the three DNA molecules using a measuring tool. They were also asked to rotate and inspect the molecules to determine left, or right-handedness. The students were asked a series of questions such as: "which helix has the smallest width?", "which helix has the largest width?", and "which 
helix is left-handed?" (see Figure 3). The next section of the lab could not be displayed until the students answered the questions correctly.

In the second section, a wildtype collagen (PDB ID: 3DMW [Boudko et al., 2008] and mutated (PDB ID: 1CAG [Bella, Eaton, Brodsky, \& Berman, 1994)]) collagen-like peptide were displayed. The mutated collagen-like peptide contained a glycine to alanine substitution in one of the repeating (X-Y-Gly) motifs resulting in a subtle unwinding of the triple helix in one region of the peptide (see Figure 5). A second difference between the two molecules was the presence of a C-terminal cysteine knot structure in the wildtype compared to the mutant. Students were asked to inspect and rotate the molecules to identify any differences, and to measure distances between several hydroxyprolines that were highlighted in the molecules. After completing the exercise, they had to answer yes/no questions such as: "is there a difference in distances between hydroxyprolines in the collagen molecules?" and "is there a difference in the ends of the two collagen molecules?"

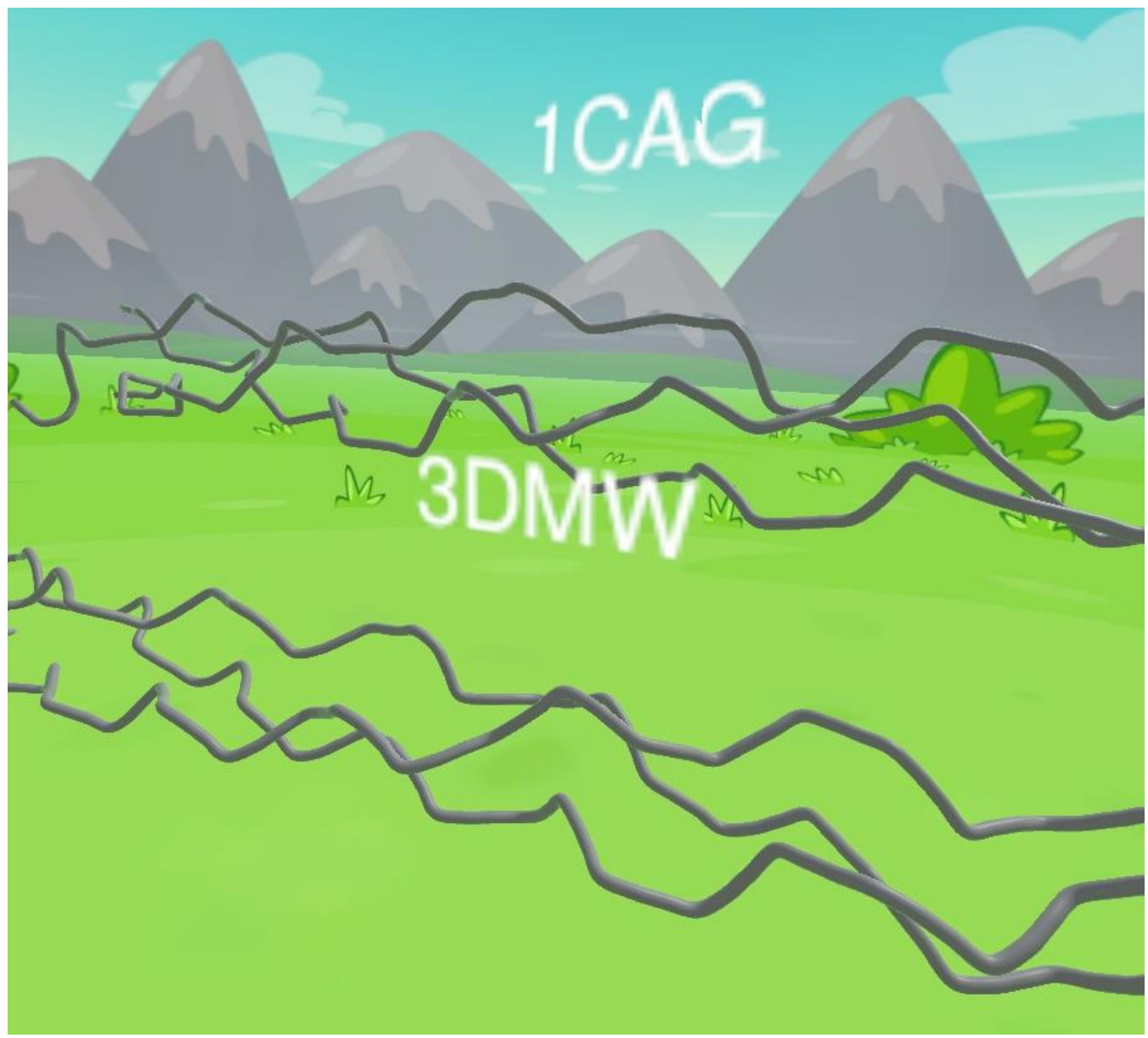

Figure 5: Collagen with Mutation. This is a wire and ribbon representation of molecules $1 \mathrm{CAG}$ and $3 \mathrm{DMW}$. There is a mutation in 1CAG which causes the unwinding present in the center of the molecule (just underneath the name tag). This unwinding is easier to see in VR.

In the third section, transcription factors TATA binding protein (PDB ID: 1TGH [Juo, et al., 1996]), sex-determining region Y (PDB ID: 1HRY [Werner, Huth, Gronenborn, \& Clore, 
1995]), integration host factor (PDB ID: 1IHF [Rice, Yang, Mizuuchi, \& Nash, 1996]), and high mobility group I-Y (PDB ID: 2EZD [Huth, et al., 1997]) bound to DNA were displayed alongside each other (see Figure 6). Students were tasked with determining if the transcription factor bound to the minor, or major groove and to note the degree of bending/twisting of the DNA structure. All four transcription factors bind to the minor groove, but students were not given this piece of information. They then had to answer three questions related to binding and bending of the DNA molecules.
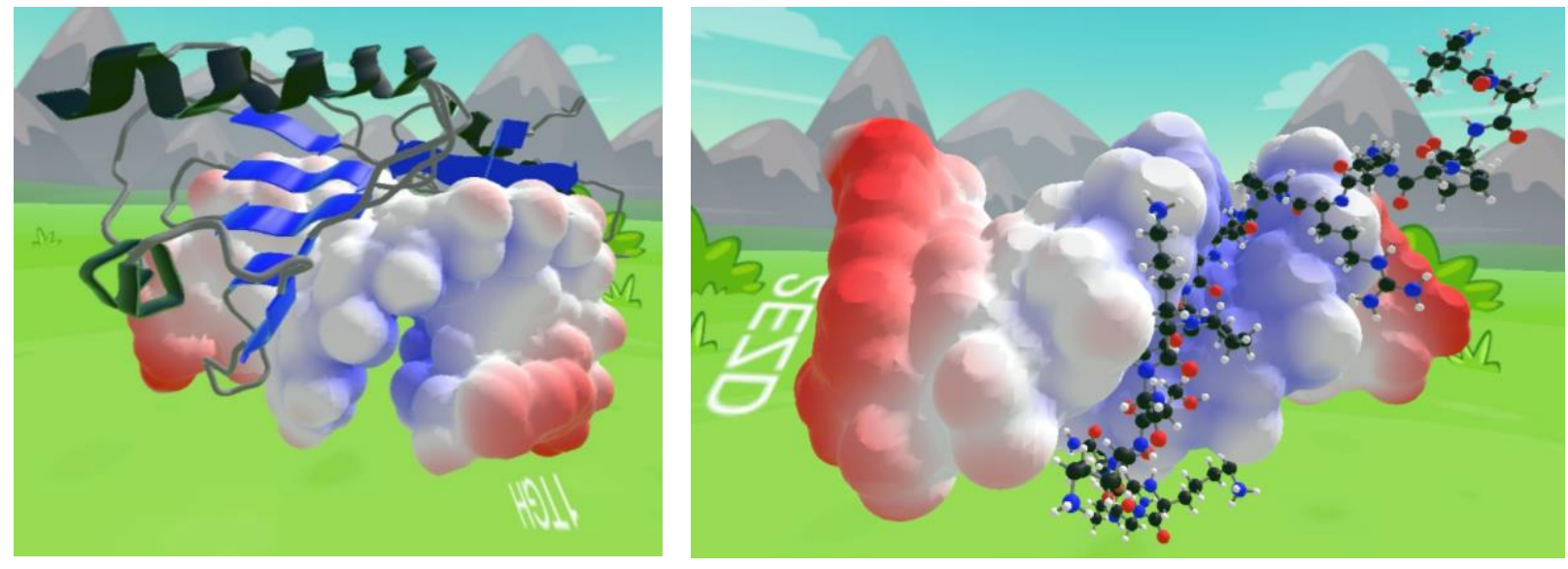

Figure 6: Hybrid View of Transcriptional Regulators. The molecule on the left is 1TGH. Here the DNA molecule is presented in surface mode while the protein has a wire and ribbon representation. The molecule on the right is 2EZD. The DNA is again presented with a surface visualization while the protein has a ball and stick presentation.

A quiz was given after the students finished the VR exercise to gauge their level of understanding. The Lab 1 quiz questions are as follows:

1. Are there differences in the major and minor groove of Z-DNA compared to B-DNA? Why are there differences?

2. Would the four transcription factors (HMG, TBP, SRY, IHF) bind better, the same, or worse to Z-DNA compared to B-DNA? What is the structural reason for the difference in binding?

3. Most collagen molecules have a X-Y-GLY repeat motif in their sequence. One of the two molecules you inspected in VR had a mutation changing GLY to ALA. This mutation can lead to the human disease Ehlers-Danlos Syndrome (connective tissue disorder). What structural differences did you notice between the two molecules (if any)? Was there a difference in helix shape? Spacing of hydroxyprolines?

Answers to these questions were graded according to the learning outcomes in Table 2 and the distribution of scores for the quiz are presented in Figure 9.

\section{Lab 2 - Hemoglobin}

Hemoglobin is one of the most widely used examples in biochemistry textbooks to underscore how quaternary protein structure is altered in response to microenvironmental fluctuations in $\mathrm{pH}$ and gaseous concentration of $\mathrm{CO} 2$ and $\mathrm{O} 2$. The structure of hemoglobin is well 
understood, which allows for thorough investigation of structure and function. The way many textbooks approach the subject is to first explain heme group structure and subsequent changes associated with oxygen binding (Berg, Tymoczko, \& Styer, 2015). Students are then introduced to the quaternary structure and led through a series of lessons/discussions to understand the effects of small fluctuations in $\mathrm{pH}$ and gases on overall hemoglobin structure. In working through this information, students are shown images of hemoglobin's quaternary structure in one of two states, the deoxy $(\mathrm{T})$ state or the oxy $(\mathrm{R})$ state. Instances of single amino acid charge changes, which are critical to $\mathrm{T}$ to $\mathrm{R}$ state transition, are shown in isolation. Although textbook explanations are well sequenced, single amino acid changes and the T/R state quaternary transitions are not well linked, which can lead to confusion in students and/or an inability for students to visualize the larger structural changes occurring in conjunction with single amino acid changes.

Key to student understanding of this material is (a) repetition of information in a variety of manners, and (b) a better method of fully integrating the details of amino acid charge fluctuations with quaternary structure. Our virtual reality lesson addresses both of these. First, the lesson is designed to illustrate the structural changes of hemoglobin in a variety of ways. Students visualize the protein in its entirety in the T state while working through a series of interactive lessons. Next, students inspect the $\mathrm{R}$ state of hemoglobin with the same lesson sequence. Working through the two states in this manner allows for repetition of content and gives students the ability to study one state in depth and then contrast what they observed with the other structural state.

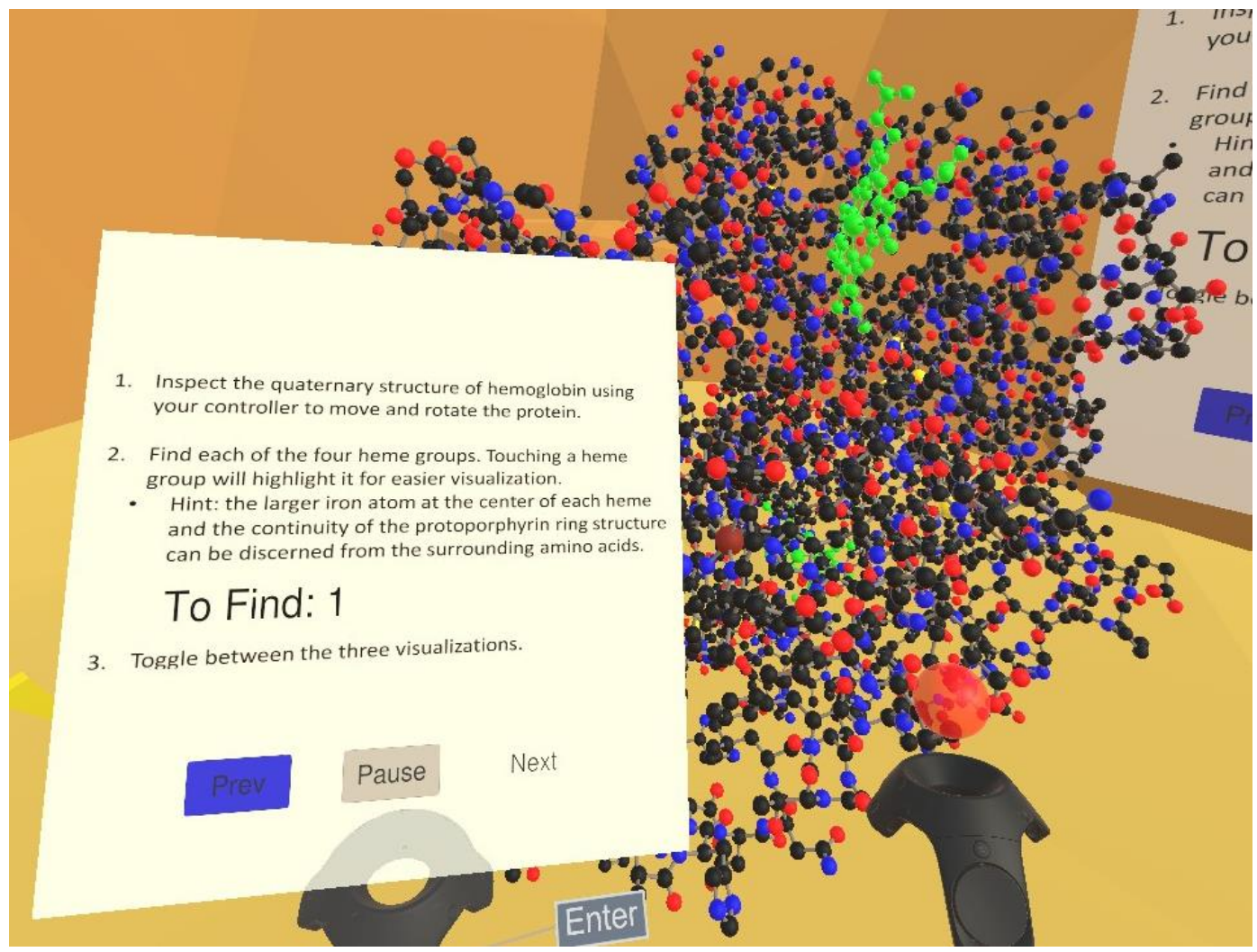

Figure 7: Finding Heme Groups. In this interactive slide students were asked to use the controller on the right to locate the 4 heme groups hemoglobin. The student has found 3 heme groups, colored green, and has one left to locate. 

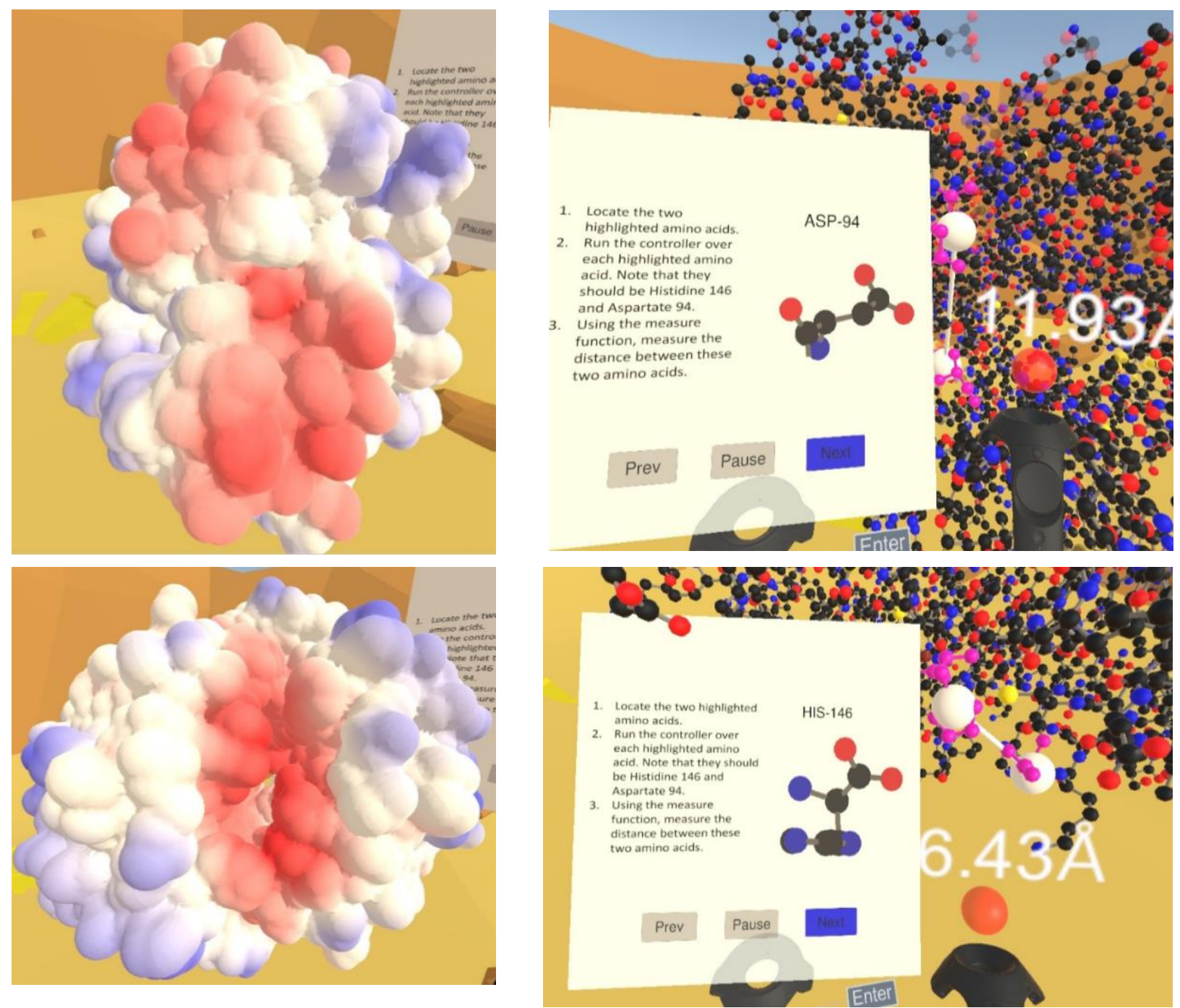

Figure 8: Deoxyhemoglobin and Oxyhemoglobin. The top row has two visualizations of the oxy (R) state of hemoglobin and the bottom has two visualizations of the deoxy (T) state. The surface representation of the $\mathrm{R}$ state (on the upper left) has no binding pocket while the $\mathrm{T}$ state (on the lower left) has a clear binding pocket. The His146 and Asp94 residues are farther apart in the $\mathrm{R}$ state (upper right) than they are in the $\mathrm{T}$ state (lower right). The measurements were made using the in-engine measuring device.

After acclimation with the program, students are tasked with first locating heme groups as is illustrated in Figure 7. They then learn to identify whether the heme is bound to oxygen simply by checking for placement of the central iron atom within the heme ring. One benefit to our lesson is that students can zoom in on a region of interest to investigate structure or measure distances between groups, while remaining within view of the entire quaternary structure. This allows students to visualize details within the larger protein structure giving them a better understanding of how small fluctuations fit within the larger structural context. After visualizing heme groups, students are tasked with identifying the central pocket, only present in the quaternary structure of deoxyhemoglobin, and visualizing positive charges surrounding this pocket. This visualization is unique to our program as many textbooks only show the pocket in isolation without charged 
regions, despite these charges being key to understanding the $\mathrm{T}$ state. Students then measure distances between amino acids that only interact with one another through salt bridges when in close proximity. In visualizing both states, students are asked to inspect key details that contrast between the states, which are responsible for either stabilization of the $\mathrm{T}$ state or maintenance of the R state. Figure 8 illustrates the differences in the central pocket between the $\mathrm{T}$ and $\mathrm{R}$ state as well as the increased distance between amino acids in the $\mathrm{R}$ state vs the same amino acids in the $\mathrm{T}$ state, as seen by the student in this lesson.

To finish the lesson, two hemoglobin proteins are displayed with a note informing students that the oxygen is not visible bound to iron in the heme-regardless of the state (oxy or deoxy). Students must use measurements or structural characteristics that they learned and practiced in the lesson to identify the correct state of hemoglobin. This repetition of learned concepts, forcing them to toggle between the overall structure and key details, reiterates key concepts required to fully understand hemoglobin function and its relationship to the deoxy and oxy states.

After finishing the lesson students were given a quiz to gauge their level of understanding. The Lab 2 quiz questions are as follows:

1. Hemoglobin has a total of oxystate can bind to heme groups; therefore, hemoglobin in the

2. How does binding of oxygen to iron affect its placement within the heme group?

a. Oxygen pulls electrons away from iron, which reduces its size allowing it fit within the plane of heme's protoporphoryn ring.

b. The smaller sized iron atom can fit between amino acids found in the beta subunits of hemoglobin.

c. The larger sized iron atom expands the heme group causing it to distort the quaternary structure of hemoglobin.

d. Iron diameter has no effect on the heme structure.

3. Hemoglobin's central binding pocket for 2,3-BPG is only present in which state (oxy or deoxystate)?

4. Binding of 2,3-BPG to the central binding pocket of hemoglobin is enhanced by the presence of what charges found on Lysine 82, Histidine 2, and Histidine 143?

5. The oxystate of hemoglobin lacks salt bridges between Histidine 146 and Aspartate 94 . How does the distance between these two amino acids change in the oxy vs deoxystate?

a. The distance between these two amino acids is much larger in the oxystate.

b. The distance between these two amino acids is much smaller in the oxystate.

c. The distance between these two amino acids does not change in either state.

6. List one way in which the oxystate of hemoglobin can be identified without looking for the presence of bound oxygen.

Answers to these questions were graded according to the learning outcomes in Table 2 and the distribution of scores for the quiz are presented in Figure 9.

\section{Procedure}

Over the course of a single semester students in either Cellular and Molecular Biology (CMB) or Biochemistry (Bio) were required to participate in both VR labs described in the previous session. After completing each lesson students were given a survey about their experience, as well as the associated quiz. Students were also asked to sign a consent form to allow the use of their survey 
and research data as part of the project (Institution IRB approved). Students who did not sign the consent form still participated in the labs, but their grade and survey data were not collected. Overall a total of 133 students signed the consent form to participate in the study. However, not every student participated in both labs and Lab 1 was completed by 128 students while Lab 2 was completed by 125 students. Additionally, there was one student who was unable to complete the labs due to nausea. As an alternative they watched an instructor walk through the lessons via a computer monitor.

The labs and quizzes were given outside of the appointed class schedule. Students in CMB or Bio would schedule a VR session using an online form. Students then participated in the VR session at the Western Carolina University VR Lab. The sessions generally took 20 minutes and were overseen by undergraduate lab assistants. The lab assistants described the study to the student, asked them to sign the participation form, and then gave them the survey and quiz. Completing the survey and quiz generally took an additional 10 minutes and the total length of each session was approximately 30 minutes. The VR lab itself contained two VR stations, each with its own PC, Vive and associated equipment. The primary benefit of this setup is that it is scalable to larger student populations by adding additional VR stations and undergraduate lab assistants. It is also compatible with distance learning programs. As long as students have access to a VR station, they can participate in the labs without the presence of the instructor.

The survey questions for each of the labs are shown in Table 1. The first 4 questions on Lab 1 and the first 5 questions on Lab 2 were all Likert Scale with the standard strongly agree to strongly disagree range of answers. The last two survey questions on each lab were open ended and were included to collect qualitative data about the student's experience.

Table 1. Lab Survey Questions.

\begin{tabular}{|l|l|}
\hline Lab 1 Question 1 & The Virtual Reality (VR) lecture helped me learn the material. \\
\hline Lab 1 Question 2 & $\begin{array}{l}\text { The three-dimensional representations of the proteins in the VR lesson helped me } \\
\text { understand the difference between A-DNA, B-DNA and Z-DNA. }\end{array}$ \\
\hline Lab 1 Question 3 & I experienced physical discomfort while participating in the VR lesson. \\
\hline Lab 1 Question 4 & I would like to participate in more VR lessons. \\
\hline Lab 1 Question 5 & $\begin{array}{l}\text { What was something about the VR lesson that was particularly helpful or } \\
\text { effective? }\end{array}$ \\
\hline Lab 1 Question 6 & $\begin{array}{l}\text { What was something about the VR lesson that was not helpful or was } \\
\text { ineffective? }\end{array}$ \\
\hline Lab 2 Question 1 & The Virtual Reality (VR) lecture helped me learn the material. \\
\hline Lab 2 Question 2 & $\begin{array}{l}\text { The three-dimensional representations of the proteins in the VR lesson helped me } \\
\text { understand the structural differences between oxy and deoxy hemoglobin. }\end{array}$ \\
\hline Lab 2 Question 3 & $\begin{array}{l}\text { Seeing proteins in three dimensions helped me understand something that I didn't } \\
\text { understand with a two-dimensional representation. }\end{array}$ \\
\hline Lab 2 Question 4 & I was able to easily read the slides during the lecture. \\
\hline Lab 2 Question 5 & I would recommend VR lessons to other students. \\
\hline Lab 2 Question 6 & $\begin{array}{l}\text { What was something about the VR lesson that was particularly helpful or } \\
\text { effective? }\end{array}$ \\
\hline Lab 2 Question 7 & $\begin{array}{l}\text { What was something about the VR lesson that was not helpful or was } \\
\text { ineffective? }\end{array}$ \\
\hline
\end{tabular}

Note: Lab 1 Questions 1-4 and Lab 2 Questions 1-5 had the 5 standard Likert scale responses: strongly agree, agree, neither agree nor disagree, disagree, and strongly disagree. Lab 1 Question 3 is the only negatively worded question. 
In addition to surveys, we also asked students to complete a quiz. The primary goal of these quizzes was not to evaluate student performance. Instead we use the quizzes to verify that students were able to understand and absorb the material presented in the corresponding lessons. The quiz questions for both labs are contained in the previous section. The labs were graded by the instructor who created the lab and the grades themselves correspond to the learning outcomes described in Table 2. The educational goal for both labs is for students to achieve at least the $61 \%$ $80 \%$ learning outcome. It is important to note that most students had not seen the content on either quiz from any other source. Because the quiz questions are so specific to the content of the lectures, we can state with confidence that students' performance on each quiz is an indicator of what they learned from the associated VR lesson. The main exception to this is that students in Bio did see the material covered by Lab 2 in class before they participated in the lab while students in $\mathrm{CMB}$ did not. For this reason, we treat Bio and $\mathrm{CMB}$ students as separate populations when analyzing the Lab 2 quiz grades.

Table 2. Lab Learning Outcomes.

\begin{tabular}{|l|l|l|}
\hline Lab & Grade & Learning Outcome \\
\hline Lab 1 & $1 \%-30 \%$ & Little, to no understanding for differences in structure of A, B, and Z-DNA. \\
\hline Lab 1 & $31 \%-60 \%$ & Some understanding for differences in structure of A, B, and Z-DNA. \\
\hline Lab 1 & $61 \%-80 \%$ & $\begin{array}{l}\text { Moderate understanding for differences in structure of A, B, Z-DNA, and } \\
\text { mode of binding of transcription factors to DNA. }\end{array}$ \\
\hline Lab 1 & $81 \%-100 \%$ & $\begin{array}{l}\text { In depth understanding for differences in structure of A, B, Z-DNA, and } \\
\text { mode of binding of transcription factors to DNA. }\end{array}$ \\
\hline Lab 2 & $1 \%-30 \%$ & $\begin{array}{l}\text { Little, to no understanding of hemoglobin structure and function in } \\
\text { relationship to oxygen binding. }\end{array}$ \\
\hline Lab 2 & $31 \%-60 \%$ & $\begin{array}{l}\text { Some understanding of hemoglobin structure and function in relationship to } \\
\text { oxygen binding. }\end{array}$ \\
\hline Lab 2 & $61 \%-80 \%$ & $\begin{array}{l}\text { Moderate understanding of hemoglobin structure and function in } \\
\text { relationship to oxygen binding. }\end{array}$ \\
\hline Lab 2 & $81 \%-100 \%$ & $\begin{array}{l}\text { In depth understanding of hemoglobin structure and function in relationship } \\
\text { to oxygen binding. }\end{array}$ \\
\hline
\end{tabular}

Note: Learning outcomes for the Lab 1 and Lab 2 quizzes corresponding to common grade ranges.

\section{Results and Data Analysis}

Both labs were delivered to students in a one semester Biochemistry (Bio) course, as well as a one semester Cellular and Molecular Biology (CMB) course as described in the previous section. Their responses and percentage quiz scores were then transcribed and anonymized. The responses for the negatively worded Lab 1 Question 3 were inverted (strongly agree swapped with strongly disagree and agree swapped with disagree) for better comparison with the other (positively worded) Likert scale questions. The responses themselves were enumerated with 1 corresponding to Strongly Disagree, 2 corresponding to Disagree, 3 corresponding to Neither Agree nor Disagree, 4 corresponding to Agree and 5 corresponding to Strongly Agree. 


\section{Lab 1 Likert Scores}

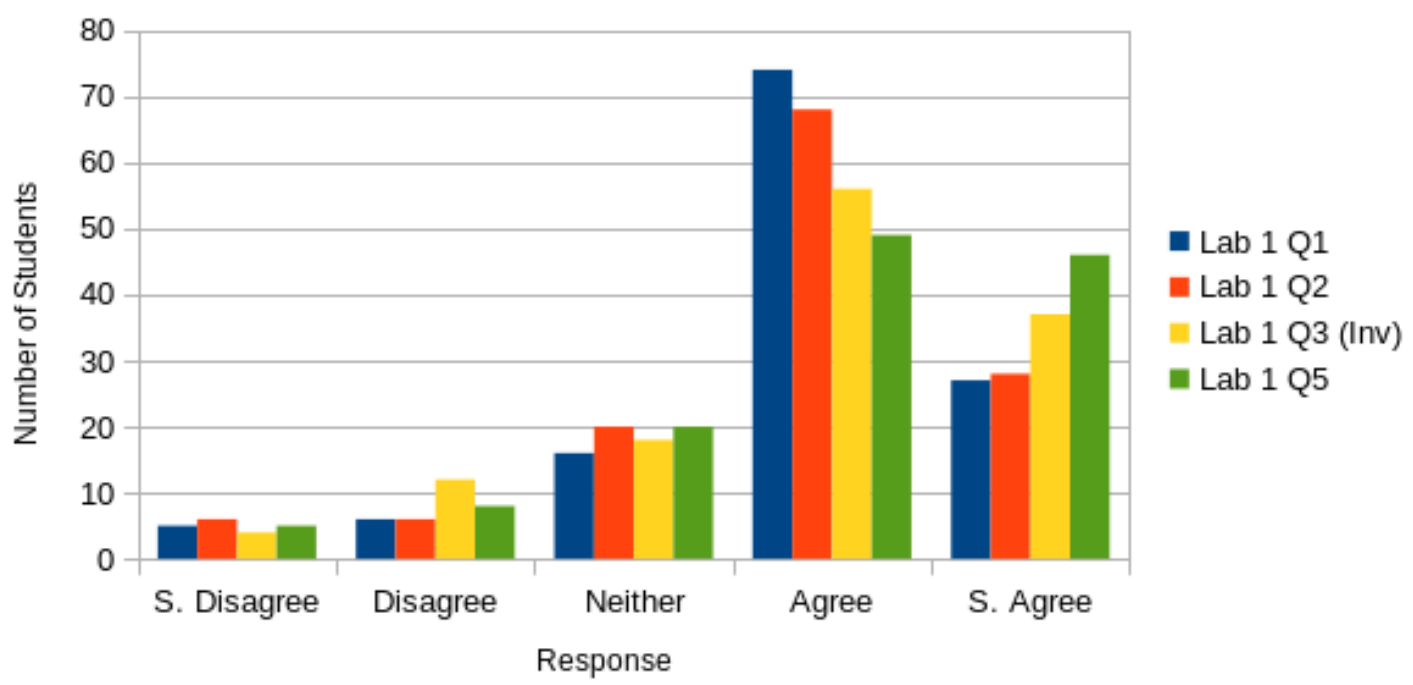

Lab 2 Likert Scores

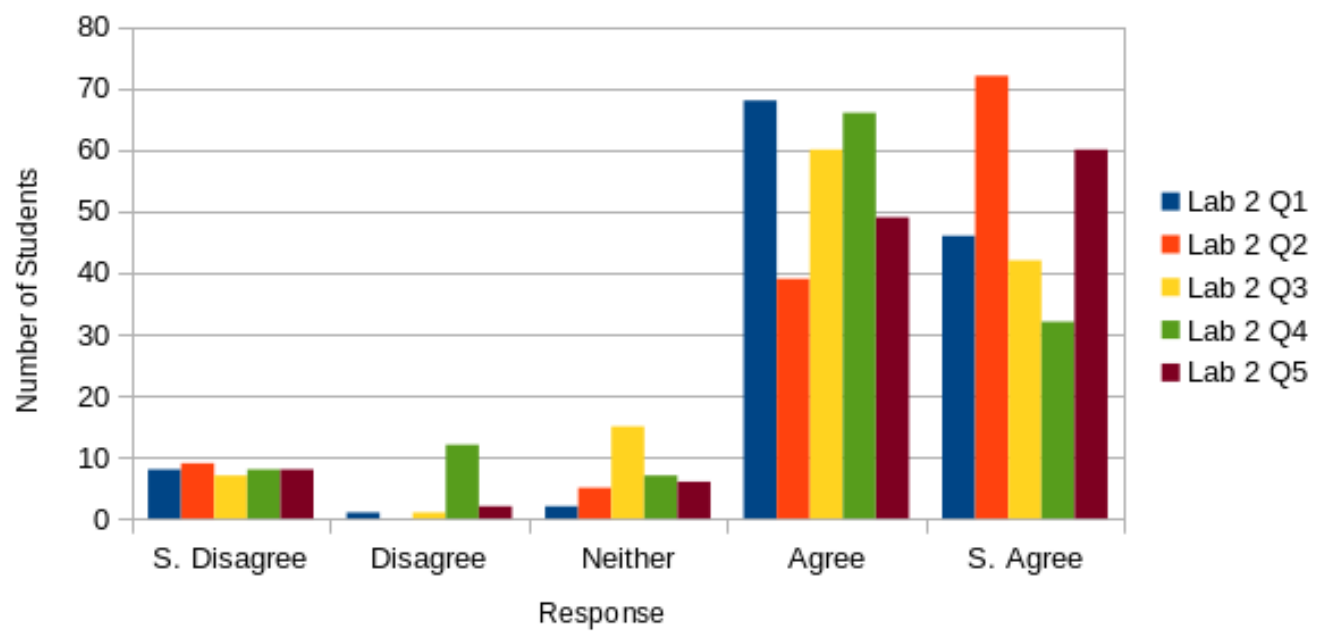

Figure 9: Likert Scale Responses for Lab 1 and Lab 2. These figures contain the distribution of responses to the Likert scale questions for the Lab 1 and Lab 2 surveys. The responses for Lab 1 Question 3 were inverted for better comparison with the other positively worded questions. The survey questions can be found in Table 1 . The clear majority of responses are either agree or strongly agree.

Overall the Likert scores for both labs were extremely positive. The distribution of responses for Lab 1 and Lab 2 can be seen in Figure 9. It is clear from the graphs that most students agreed or strongly agreed with the positively worded statements that VR helped them learn the material, that it helped them understand material they didn't understand otherwise, and that they would participate in more VR lessons and would recommend them to others. Not only do students like the VR labs, but they feel that the VR visualizations help them to better understand the content. Table 3 contains the statistics for the Lab 1 and Lab 2 survey responses. Specifically, Table 3 contains the mean and mode for each response, as well as the standard deviation, number of 
responses, and one-sided p-value for the sign test. The p-value is computed using the binomial distribution according to the alternative hypothesis that students were more likely to choose Strongly Agree/Agree than Strongly Disagree/Disagree, ignoring responses of Neither. A small pvalue indicates that on average students who did not answer Neither Agree Nor Disagree were more likely to give a positive response than a negative one. The scores for every survey question were very high. Each question had an average score of 3.8 or greater and the mode for each question was either 4 (Agree) or 5 (Strongly Agree). The results are all statistically significant with very small p-values.

Table 3. Statistics for Lab Survey Questions

\begin{tabular}{|c|c|c|c|c|c|}
\hline Question & Mean & Mode & Std. Dev. & N & p-value \\
\hline Lab 1 Question 1 & 3.88 & 4 & 0.93 & 128 & $<.00001$ \\
Lab 1 Question 2 & 3.83 & 4 & 0.98 & 128 & $<.00001$ \\
Lab 1 Question 3 & 3.87 & 4 & 1.04 & 128 & $<.00001$ \\
Lab 1 Question 4 & 3.96 & 4 & 1.06 & 128 & $<.00001$ \\
Lab 2 Question 1 & 4.14 & 4 & 0.99 & 125 & $<.00001$ \\
Lab 2 Question 2 & 4.32 & 5 & 1.08 & 125 & $<.00001$ \\
Lab 2 Question 3 & 4.03 & 4 & 1.00 & 125 & $<.00001$ \\
Lab 2 Question 4 & 3.82 & 4 & 1.12 & 125 & $<.00001$ \\
Lab 2 Question 5 & 4.21 & 5 & 1.06 & 125 & $<.00001$ \\
\hline
\end{tabular}

Note: This table contains the mean and mode for answers to the Likert Scale survey questions with 1 corresponding to strongly disagree and 5 corresponding to strongly agree. The responses for Lab 1 Question 3 were inverted for better comparison with the other positively worded questions. The table also indicates the number of students who participated in the survey as well as the p-value for the one-sided sign test corresponding to the alternative hypothesis that students were more likely to choose Strongly Agree/Agree than Strongly Disagree/Disagree, ignoring responses of Neither.

It is interesting to note that the scores for the second lab are even higher than the first lab, particularly in the case of questions 1 and 2. Question 1, "The Virtual Reality (VR) lecture helped me learn the material," was the same for both labs and Question 2 was the same in that it asked students if the VR lesson helped the student understand the specific content of the lab. The mean score for question 1 increased from 3.88 to 4.14 between Lab 1 and Lab 2. Specifically, 37\% of students had a stronger agreement with Question 1 on Lab 2 than Lab 1 and $87 \%$ agreed at least as strongly for Lab 2 as Lab 1. Similarly, the mean score for Question 2 increased from 3.83 to 4.32 from Lab 1 to Lab 2. Here 51\% of students had a stronger agreement in the second lab and $88 \%$ agreed at least as strongly. This increase in positive responses is consistent with the hypothesis that the positive response to the VR lessons was not due to their novelty. If this were the case, we would have expected scores to be lower for the second lab. Based off observational data and students' open-ended responses, the increase in scores is likely due to their increased familiarity with the system. They spent less time learning the controls and more time engaging with the material. 


\section{Lab Quiz Scores}

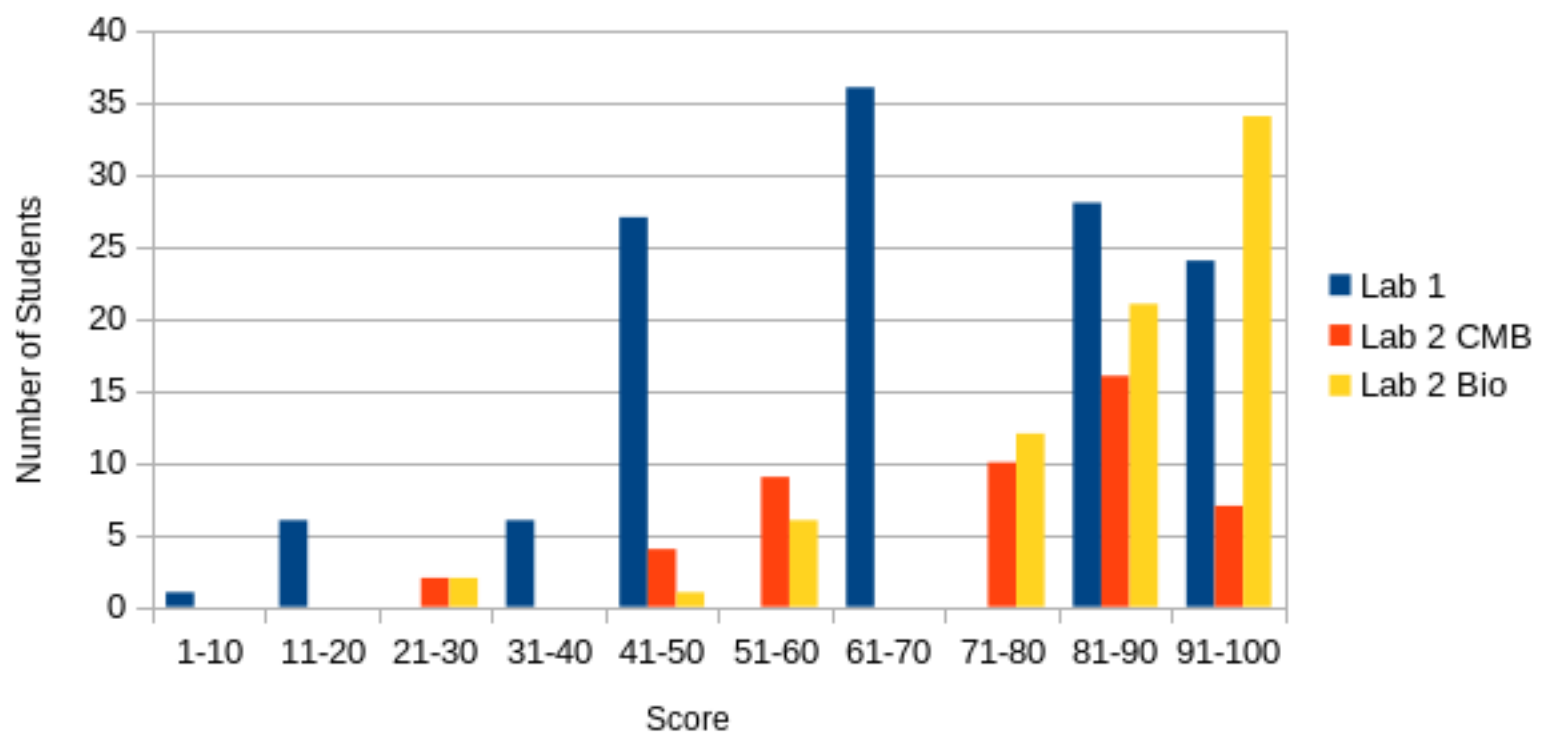

Figure 10: Percentage Lab Scores. The chart indicates the distribution of quiz scores for Lab 1, and the two subpopulations of Lab 2. Lab 2 CMB is the subpopulation of students in Cellular and Molecular Biology who did not see the lab material in class. Lab 2 Bio is the subpopulation of students in Biochemistry who did see the lab material in class before taking the lab.

In addition to the survey data we also collected and analyzed students' percentage scores on the quiz portion of the labs. Students were assigned these scores by the instructor who created the lab according to the rubric in Table 2. None of the students who took Lab 1 had seen the material presented in the lab in class. On the other hand, there were two subpopulations for Lab 2. Students in CMB did not see the material in the lab as part of their regular coursework, while students in Bio studied the lab material in class prior to taking the lab. For this reason, we analyze the Lab 2 grades from these populations separately. The distribution of scores for Lab 1 and the two subpopulations of Lab 2 is shown in Figure 10. Table 4 contains the average grade, median grade, standard deviation, number of responses, and p-value for the same three data sets.

Table 4. Lab Quiz, Grades

\begin{tabular}{|l|c|c|c|c|c|}
\hline $\begin{array}{l}\text { Quiz } \\
\text { Population }\end{array}$ & $\begin{array}{c}\text { Average } \\
\text { Grade }\end{array}$ & $\begin{array}{c}\text { Median } \\
\text { Grade }\end{array}$ & Std. Dev. & N & p-value \\
\hline Lab 1 & $68.62 \%$ & $66.67 \%$ & 23.07 & 128 & .00001 \\
Lab 2 Bio & $84.42 \%$ & $85.71 \%$ & 19.82 & 77 & $<.00001$ \\
Lab 2 CMB & $73.51 \%$ & $71.43 \%$ & 19.32 & 48 & $<.00001$ \\
\hline
\end{tabular}

Note: This table contains the average, median, and standard deviation of the percentage quiz grades for Lab 1 and both subpopulations of Lab 2. Lab 2 CMB is the subpopulation of students in Cellular and Molecular Biology who did not see the lab material in class. Lab 2 Bio is the subpopulation of students in Biochemistry who did see the lab material in class before taking the lab. The table also indicates the number of students in each population as well as the (onesided) t-test p-value corresponding to the alternative hypothesis $\mu>60 \%$ 
First, note that the quiz questions for Lab 1 were significantly less granular than those for Lab 2, which is why the Lab 1 scores are more tightly clustered. The average grade for Lab 1 was $69 \%$ and has a roughly normal distribution. The average and median grade for Lab 1 both correspond to the educational objective that students have a "Moderate understanding for differences in structure of A, B, Z-DNA, and mode of binding of transcription factors to DNA." The result is significant with a one tailed t-test $\mathrm{p}$-value of .00001 corresponding to the alternative hypothesis $\mu>60 \%$. We conclude from this, as well from our subjective observations, that Lab 1 generally meets its educational objectives. Students earned solid scores with a traditional grade distribution. We do not make any claims about the general effectiveness of VR as a teaching tool and formal educational research would be needed to determine the precise educational impact of VR on student learning.

The grade data for Lab 2 is more complex. The average Lab 2 grade for students in CMB was $74 \%$ while the average Lab 2 grade for students in Bio was $84 \%$. The grades for the CMB population were roughly normally distributed while the grades for the Bio population are skewed to the right. The difference in the averages and distributions for the Lab 2 sub-populations is likely because students in $\mathrm{CMB}$ did not see the lab material in class while students in Bio did see the material. On average, students in CMB achieved the educational objective of having a "Moderate understanding of hemoglobin structure and function in relationship to oxygen binding." The result is significant with a one tailed t-test $p$-value of less than 0.00001 corresponding to the alternative hypothesis $\mu>60 \%$. In general, the Lab 2 outcomes for CMB students were similar to the Lab 1 outcomes in that the students earned good scores with a traditional grade distribution. On the other hand, students in Bio performed significantly better on the second lab than their counterparts in CMB. On average they achieved the educational objective of having an "In depth understanding of hemoglobin structure and function in relationship to oxygen binding." The result is significant with a one tailed t-test $p$-value of .02 corresponding to the alternative hypothesis $\mu>80 \%$. The high scores and right skewed distribution are indicative of high performing students. The fact that students who saw the material in class received on average better scores than those who did not would indicate that VR is not a sufficient replacement for traditional educational techniques. Furthermore, we did not test students' knowledge before they participated in the lab so we cannot say definitively if the VR lesson increased their understanding of the material. However, there are a number of potential pitfalls with the in-class presentation of hemoglobin, as discussed earlier. Moreover, the most positive survey answers for the entire study were in response to the question "The three-dimensional representations of the proteins in the VR lesson helped understand the structural differences between oxy and deoxy hemoglobin," with an average score of 4.32 and a mode of "Strongly Agree." With this in mind, we take the high quiz scores by students in Bio as a positive sign that the VR lesson benefited student understanding. Overall, while we cannot make any direct claims about the effectiveness of VR as a teaching tool, we can reasonably state that Lab 2 satisfied its educational objectives.

\section{Conclusions and Discussion}

There are several conclusions we can draw from this experience. The first is that students really liked the VR labs and felt that the VR visualizations improved their understanding of the material. Survey responses for the labs were extremely high, and this positive response is backed up by observational evidence as well as the open-ended survey responses. Students found the VR lessons helpful and their engagement with the lectures seemed to increase over time as they got used to the format and control schemes. While a strong positive response can be reason enough to use an 
educational tool, the VR labs also achieved their learning objectives. The quiz grades were reasonably good considering the material was delivered via a 20-minute recorded lecture. What is more, students who experienced the material both in VR and in class scored very well on the quizzes. More research would need to be done to precisely determine if the VR lab increased the students understanding beyond what they had achieved in class. However, considering that providing quality $3 \mathrm{D}$ visualizations of molecules to students is a long running challenge, as well as the extremely positive response to the survey questions asking if the VR lectures helped students understand the material, we consider these high scores to be a positive result. Put together, the positive student response and learning outcomes mean that this project has been a success from an educational perspective.

The project has also been a success from a technical perspective. Over 200 VR sessions were delivered to students over 14 weeks, supervised only by undergraduate lab assistants. The lab sessions ran smoothly without any significant technical assistance with only the occasional bug or glitch. The setup used for these labs is scalable to larger courses with multiple sections and is generally ready for everyday use. Furthermore, this lab setup is compatible with distance learning programs. As long as the students have access to a VR Lab, instructors are not required for students to participate in the lessons. The presentation platform itself could use more refinement. Creating VR labs still requires knowledge of the Unity platform. While the setup is friendly enough that, with some assistance, a technically proficient undergraduate was able to create a VR lesson for an undergraduate project, it is not robust enough for the average biology or chemistry instructor. More work needs to be done streamlining and polishing the lecture creation process. Additionally, while the molecule visualization package performed admirably, it could also use further testing and refinement so that it can reliably provide the same quality of visualization as more developed molecular modelling software. In general, there is a real need for professional grade educational VR tools, especially modular tools to allow instructors to quickly assemble labs and lessons without in depth knowledge of 3D computer visualization.

A number of open questions remain, both technical and educational. More work still needs to be done to formally measure the educational impact of VR lectures, as well as discovering best practices and techniques. For instance, the visualizations in these lessons were intentionally made to resemble those found in standard visualization tools. However, there might be an educational benefit to rendering the proteins on different scales or from different perspectives. Would students benefit from seeing an ion channel on the same scale as an ion, for instance? Other open questions include studying how these tools and techniques could be adapted to Augmented Reality, where the ability to overlay realistic 3D molecular visualizations on the real world opens up exciting new possibilities. For example, students could use augmented reality to interact with virtual 3D molecular visualizations while also working in groups, taking notes, and generally participating in traditional educational activities (Berry \& Board, 2014).

\section{References}

Bella, J., Eaton, M., Brodsky, B., \& Berman, H. (1994). Crystal and molecular structure of a collagen-like peptide at 1.9 A resolution. Science, 266(5182), 75-81.

https://doi.org/10.1126/science.7695699

Berg, J. M., Tymoczko, J. L., \& Styer, L. (2015). Biochemistry (8th ed.). New York, NY: W. H. Freeman \& Company. 
Berry, C., \& Board, J. (2014). A Protein in the palm of your hand through augmented reality. Biochemistry and Molecular Biology Education, 42(5), 446-449.

https://doi.org/10.1002/bmb.20805

Borrel, A., \& Fourches, D. (2017). RealityConvert: a tool for preparing 3D models of biochemical structures for augmented and virtual reality. Bioinformatics, 33(23), 3816-3818.

https://doi.org/10.1093/bioinformatics/btx485

Boudko, S., Engel, J., Okuyama, K., Mizuno, K., Bachinger, H., \& Schumacher, M. (2008). Crystal structure of human type III collagen Gly991-Gly1032 cystine knot-containing peptide shows both 7/2 and 10/3 triple helical symmetries. Journal of Biological Chemistry, 283(47), 32580-32589. https://doi.org/10.1074/jbc.M805394200.

Garcia-Bonete, M.-J., Jensen, M., \& Katona, G. (2019). A practical guide to developing virtual and augmented reality exercises for teaching structural biology. Biochemistry and Molecular Biology Education, 47(1), 16-24. https://doi.org/10.1002/bmb.21188

Garzón, J. C., Magrini, M. L., \& Galembeck, E. (2017). Using augmented reality to teach and learn biochemistry. Biochemistry and Molecular Biology Education, 45(5), 417-420.

https://doi.org/10.1002/bmb.21063

Goehle, G. (2018). Teaching with Virtual Reality: Crafting a Lesson and Student Response. International Journal for Technology in Mathematics Education, 25(1), 35-45. https://doi.org/10.1564/tme_v25.1.04

Hardwick, J., Ptchelkine, D., El-Sagheer, A., Tear, I., Singleton, D., Phillips, S., Lane, A., \& Brown, T. (2017). 5-Formylcytosine does not change the global structure of DNA. Nature Structural \& Molecular Biology, 24(6), 544-552. https://doi.org/10.1038/nsmb.3411

Huth, J., Bewley, C., Nissen, M., Evans, J., Reeves, R., Gronenborn, A., \& Clore, G. (1997). The solution structure of an HMG-I(Y)-DNA complex defines a new architectural minor groove binding motif. Nature Structural Biology, 4(8), 657-665. https://doi.org/10.1038/nsb0897-657

Johnston, A. P., Rae, J., Ariotti, N., Bailey, B., Lilja, A., Webb, R., . . Parton, R. (2018). Journey to the centre of the cell: Virtual reality immersion into scientific data. Traffic, 19(2), 105-110. https://doi.org/10.1111/tra.12538

Juo, Z., Chiu, T., Leiberman, P., Baikalov, I., Berk, A., \& Dickerson, R. (1996). How proteins recognize the TATA box. Journal of Molecular Biology, 261(2), 239-254. https://doi.org/10.1006/jmbi.1996.0456

Kaphingst, K. A., Persky, S., Mccall, C., Lachance, C., Beall, A. C., \& Blascovich, J. (2009). Testing Communication Strategies to Convey Genomic Concepts Using Virtual Reality Technology. Journal of Health Communication, 14(4), 384-399. https://doi.org/10.1080/10810730902873927

Lam, W. W., \& Siu, S. W. (2017). PyMOL mControl: Manipulating molecular visualization with mobile devices. Biochemistry and Molecular Biology Education, 45(1), 76-83.

https://doi.org/10.1002/bmb.20987

Luo, Z., Dauter, M., \& Dauter, Z. (2014). Phosphates in the Z-DNA dodecamer are flexible, but their PSAD signal is sufficient for structure solution. Acta Crystallographica, 70(7), 1790-1800. https://doi.org/10.1107/S1399004714004684

Mikropoulos, T. A., Katsikis, A., Nikolou, E., \& Tsakalis, P. (2003). Virtual environments in biology teaching. Journal of Biological Education, 37(4), 176-181. https://doi.org/10.1080/00219266.2003.9655879

Portin, P. (2014). The birth and development of the DNA theory of inheritance: sixty years since the discovery of the structure of DNA. Journal of Genetics, 93(1), 293-302. https://doi.org/10.1007/s12041-014-0337-4

Rice, P., Yang, S., Mizuuchi, K., \& Nash, H. (1996). Crystal structure of an IHF-DNA complex: a proteininduced DNA U-turn. Cell, 87(7), 1295-1306. https://doi.org/10.1016/s0092-8674(00)81824-3

Shui, X., Mcfail-Isom, L., Hu, G., \& Williams, L. (1998). The B-DNA dodecamer at high resolution reveals a spine of water on sodium. Biochemistry, 37(23), 8341-8355. https://doi.org/10.1021/bi973073c 
Ussery, D. W. (2002). DNA structure: A-, B- and Z-DNA helix families. Chichester, England: John Wiley \& Sons. https://doi.org/10.1038/npg.els.0003122

Werner, M., Huth, J., Gronenborn, A., \& Clore, G. (1995). Molecular basis of human 46X,Y sex reversal revealed from the three-dimensional solution structure of the human SRY-DNA complex. Cell, 81(5), 705-714. https://doi.org/10.1016/0092-8674(95)90532-4

\section{$\pi \mathrm{L}$}

